Ultra-fast spin-mixing in a diketopyrrolopyrrole monomer / fullerene blend charge transfer state

Enrico Salvadori, $¥$ Nathaniel Luke, $\ddagger$ Jordan Shaikh, Anastasia Leventis, Hugo Bronstein, Christopher W.M. Kay, and Tracey M. Clarke, ${ }^{*}$

Diketopyrrolopyrrole (DPP) is one of the most common building blocks for small molecules and conjugated polymers designed for organic electronic applications. By attaining a detailed understanding of the photophysical behaviour for a simple DPP-based molecule in fullerene blends, we establish a foundation for spectroscopic investigations into more complex DPP-based systems. Transient absorption spectroscopy (TAS) and time-resolved electron paramagnetic resonance (TREPR) spectroscopy are used to examine bulk heterojunction blend films of a small diketopyrrolopyrrole-based molecule, 2,5-bis(2-hexyldecyl)-3,6-di(thiophen-2-yl)-2,5dihydropyrrolo[3,4-c]pyrrole-1,4-dione (TDPP) with the common fullerene derivatives [6,6]-phenylC61-butyric acid methyl ester (PC60BM) and [6,6]-phenyl-C71-butyric acid methyl ester (PC70BM). Following pulsed laser excitation, the spectral signatures of a fullerene anion and a TDPP triplet state are observed on the picosecond timescale by TAS. The presence of these species imply the formation of a TDPP:PCBM charge transfer state that subsequently undergoes ultra-fast spin-mixing and geminate recombination to produce a TDPP triplet state. The overall photophysical mechanism is confirmed by TR-EPR spectroscopy, which unambiguously shows that the TDPP triplet is formed via spin-mixing in the TDPP:PCBM charge transfer state, rather than direct intersystem crossing from the excited singlet state.

Introduction

Diketopyrrolopyrrole (DPP) is one of the most commonly used moieties in organic electronics. ${ }^{1,2}$ DPP combines excellent stability, considerable synthetic versatility, high absorption coefficient, and strong fluorescence. Furthermore, it has produced high efficiencies in a number of organic electronic devices, including organic photovoltaics, light emitting diodes, and transistors. A key advantage of DPP is that it can be employed in both small molecule and conjugated polymer applications: in bulk heterojunction polymer solar cells, for example, efficiencies of over $9 \%^{3-5}$ have been measured, while efficiencies of over $7 \%^{6}$ have been achieved with DPP-based small molecules.

Despite its popularity, few reports exist in the literature regarding small molecule DPP's fundamental photophysics in a bulk heterojunction environment with fullerene. The photophysical mechanisms present in an organic solar cell have a direct bearing on its efficiency. The short circuit current is directly proportional to the charge carrier density present in the device, while the open circuit voltage is correlated with the charge transfer (CT) state energy, and the fill factor is strongly affected by bimolecular recombination. Of particular concern is the potential formation of triplet states. If the triplet state energy - of the donor or the fullerene - is lower than the energy of the CT state, then formation of a triplet state can occur instead of the desired separated charges, which creates an additional loss pathway and reduces the device efficiency. This has been observed in a number of conjugated polymer/fullerene blends, and has often been speculated to occur via spin-mixing in the charge transfer state..$^{7-9}$

An experimental technique that has proven extremely useful for investigating the photophysical behaviour of organic photovoltaic blends is transient absorption spectroscopy (TAS). TAS directly 
monitors the optical absorption of photogenerated transient species, such as charge carriers and, excited singlet and triplet states. TAS provides information on the identity, yield and recombination of these transient species. It has been applied to numerous polymer:blend systems, ${ }^{10-19}$ where the charge carrier decay kinetics typically exhibit a power law decay $\left(\triangle \mathrm{OD} \propto \mathrm{t}^{-\alpha}\right)$. This power law behaviour is consistent with models describing bimolecular recombination of dissociated charge carriers in the presence of an exponential distribution of localised (trapped) states, ${ }^{20}$ and is very different from the behaviour expected of triplet states. Triplet state relaxation back to the ground state is a first order process (at low excited state concentrations), which manifests as a monoexponential decay in the transient absorption kinetics.

An additional spectroscopic technique that is highly complementary to TAS is time-resolved electron paramagnetic resonance (TR-EPR) spectroscopy, a techniques it that can offer critical mechanistic details. Owing to its unique sensitivity to paramagnetic species, TR-EPR can detect and assign triplet states and discriminate them from charge transfer states and radical pairs. The shape of the TR-EPR spectra of organic triplets reports on the mechanism of formation of the paramagnetic species, as it is directly dependent on the difference in populations of the three triplet sublevels. A common feature of all mechanisms leading to triplet formation is spin-selectivity. This translates into distinctive non-equilibrium population of spin sub-levels (a phenomenon known as spin polarisation), which can be used to pinpoint energy transfer pathways and charge transfer processes.

Previous transient spectroscopic studies of DPP-based small molecules have concentrated on the pristine DPP molecule with ancillary conjugated rings (thiophenes, for example), ${ }^{21,22}$ or DPP as one component of a covalently bound triad system. ${ }^{23,24}$ Remarkable results have already been observed: for example, pristine TDPP (DPP with two ancillary thiophene rings) has been reported to show singlet fission in thin films. ${ }^{25}$ However, to our knowledge, no time-resolved spectroscopic study has been performed on simple DPP monomers in a bulk heterojunction film blended with fullerene. Furthermore, previous TAS studies have typically examined spectral features in the visible region only.

It is the aim of this paper, therefore, to build on previous literature work by examining TDPP in bulk heterojunction blend films with fullerenes, with particular focus on transient absorption spectral features in the near-infrared region. Importantly, TR-EPR spectroscopy is also used to complement and validate the TAS results, providing insight into the photophysical mechanisms present. The motivation is to establish a baseline of photophysical behaviour that will assist spectroscopic investigations into more complex DPP-based molecules blended with fullerenes, in order to enhance the efficiency of organic electronic applications. A TDPP:fullerene weight ratio of 1:2 was used, due to the high power conversion efficiencies achieved using this ratio, ${ }^{4}$ although it should be noted that the optimum ratio varies with different DPP systems. For this reason, TAS was also measured for a 1.6:1 blend for comparison.

It was found in this paper that a TDPP:fullerene charge transfer state forms on ultra-fast timescales $(<100 \mathrm{fs})$. This CT state undergoes spin-mixing and geminate recombination to form the TDPP triplet 
state, again on ultra-fast timescales. The CT state was clearly observed via a distinct fullerene anion transient absorption spectral signature with a monoexponential decay lifetime of $200-500$ ps, depending on the identity of the fullerene. Furthermore, the TAS shows a rise in the TDPP triplet population, peaking at approximately $30-40 \mathrm{ps}$. This rise in TDPP triplet population with the concurrent decay of the fullerene anion is further evidence that the fullerene is part of a TDPP:fullerene CT state that is undergoing spin-mixing, followed by recombination to form the TDPP triplet. This interpretation was confirmed through the use of TR-EPR spectroscopy, which unequivocally shows that the TDPP triplet has been formed through a spin-mixing CT state mechanism.

Results

Steady state absorbance and fluorescence

The structure of TDPP is shown in the inset of Figure 1. Pristine diketopyrrolopyrrole-based monomer (TDPP) films show a maximum absorbance at $510 \mathrm{~nm}$, with a shoulder observed at 490 and a smaller peak at $565 \mathrm{~nm}$ (Figure 1). Addition of fullerene causes an increase in the relative amplitude of the shoulder at $565 \mathrm{~nm}$ (Figure S1a, supporting information). For polymer/fullerene blends this observation is usually linked to an enhancement in crystallinity. ${ }^{26}$ If this holds true for the TDPP:PCBM films as well, this implies that the presence of the fullerene molecules imposes some degree of ordering on the TDPP molecules. In turn this could suggest the formation of disordered aggregates in the pristine DPP film. This is supported by the dilute solution state absorbance spectra, which show this lowest energy band (shifted to $550 \mathrm{~nm}$ ) to be the highest in relative amplitude.

The pristine TDPP films fluoresce with a peak maximum at $690 \mathrm{~nm}$. The TDPP fluorescence is almost completely quenched by the addition of fullerene, with quenching rates of $97 \%$ observed for both TDPP:PC60BM and TDPP:PC70BM (Figure S1b, supporting information). This typically suggests quenching of the donor exciton via electron or energy transfer to the fullerene.

\section{Picosecond transient absorption spectroscopy}

The ps-TA spectrum of the pristine TDPP film shows two strong peaks at approximately $800 \mathrm{~nm}$ and $1600 \mathrm{~nm}$ (Figure 2a). At low excitation densities, both peaks decay mono-exponentially with the same lifetime of $(150 \pm 10) \mathrm{ps}$, suggesting they arise from the same transient species (Figure $2 \mathrm{~b}$ ). The ultra-fast generation of these peaks, first order decay kinetics, and lifetime are consistent with a singlet state, and both bands can therefore be attributed to the TDPP singlet exciton. The loss of mono-exponential behaviour at higher excitation densities can be assigned to singlet-singlet annihilation. The $800 \mathrm{~nm}$ band (or the edge of it, in visible region only studies) has been observed before in TAS studies of related DPP-based molecules and was also assigned to the $S_{1}$ state. ${ }^{21,23,24}$ Interestingly, there is also evidence of very weak, broad, longer-lived bands at $850-1100 \mathrm{~nm}$ that remain after the strong singlet peaks have decayed. Extrapolating the decay of these additional peaks to longer times shows that these signals will have completely decayed by $500 \mathrm{~ns}$, and this explains their absence in the $\mu$ s-TAS data (vide infra). The identity of this feature is unclear: it is possible that it can be assigned to short-lived triplets that form inefficiently via direct ISC. 
The ps-TA spectrum of TDPP:PC60BM is shown in Figure 2c. The two TDPP singlet exciton peaks are evident at the earliest times, but they are accompanied by additional features at $870 \mathrm{~nm}$ and 1030 $\mathrm{nm}$. The $1030 \mathrm{~nm}$ band has completely decayed by the end time resolution (6 ns), but the $870 \mathrm{~nm}$ band persists beyond this time. Although the $870 \mathrm{~nm}$ band is immediately apparent at the earliest times ( $<100 \mathrm{fs}$ ), at low excitation densities a growth in the signal amplitude is observed (see Figure $2 \mathrm{~d}$ ), with the maximum occurring at approximately $40 \mathrm{ps}$. This growth is not observed at higher excitation densities, suggesting that the transient species may be quenched by higher order processes. The $1030 \mathrm{~nm}$ band position is consistent with the PC60BM anion, and at low excitation densities it decays with mono-exponential behaviour and a lifetime of ( $230 \pm 40)$ ps. This lifetime was determined using a probe wavelength of $1120 \mathrm{~nm}$, on the red edge of the PCBM anion peak in order to avoid contamination from the longer lived $870 \mathrm{~nm}$ band. The mono-exponential decay of the PC60BM anion and hundreds of picosecond lifetime indicates that it is decaying via geminate recombination.

The assignment of the $1030 \mathrm{~nm}$ band to the PC60BM anion received further support when the ps-TA spectrum of TDPP:PC70BM was measured (Figure 3e). In the latter case, the $1030 \mathrm{~nm}$ band was absent: instead, a band was observed at approximately $1320 \mathrm{~nm}$. The position of this band is consistent with literature values for the PC70BM anion. ${ }^{27,}{ }^{28}$ Mono-exponential decay dynamics were observed again, with a lifetime of $(420 \pm 80)$ ps (measured from the blue edge, at $1200 \mathrm{~nm}$ ). This lifetime is longer than that observed for the PC60BM anion. As with the TDPP:PC60BM blend, a long lived peak was observed at $870 \mathrm{~nm}$ in the TDPP:PC70BM film. The rise in population of this $870 \mathrm{~nm}$ band is more apparent for TDPP:PC70BM (Figure 2f), with the peak population occurring at approximately $30 \mathrm{ps}$.

Considering that the long-lived $870 \mathrm{~nm}$ band does not shift when the fullerene is changed, this means that it can be assigned to a TDPP species. As such, the $870 \mathrm{~nm}$ band is most likely either the TDPP triplet or the TDPP radical cation. The lack of similarity between the kinetics of the fullerene anion decay and the $870 \mathrm{~nm}$ band imply that it is not the TDPP radical cation: one would expect identical kinetics of geminate recombination between cation and anion. However, it should also be pointed out that the DPP radical cation absorbs at $750 \mathrm{~nm}$ in solution ${ }^{22}$ so the possibility that the 870 $\mathrm{nm}$ band is related to the DPP cation cannot be entirely ruled out solely on the basis of these data.

Assuming that the $870 \mathrm{~nm}$ band is indeed the TDPP triplet, the ps-TAS results therefore suggest the following mechanism. Initial excitation of the TDPP produces TDPP singlet excitons, which then undergo an ultra-fast electron transfer to the fullerene, forming a TDPP/fullerene charge transfer state. Spin-mixing (which also must occur on ultra-fast timescales) then follows, producing the triplet CT state. The triplet CT state then undergoes geminate recombination to form the TDPP triplet (the lowest energy triplet state in the system). The fullerene anion peak can therefore most likely be attributed to the CT state rather than free fullerene anions, since the increase in the triplet population occurs concurrently with the fullerene anion decay. Furthermore, the rise in triplet population on the picosecond timescale is additional support that a spin-mixing $\mathrm{CT}$ state mechanism is active, since direct ISC is typically a much slower process. Furthermore, the existence of DPP:fullerene CT states have been proposed in a number of reports. ${ }^{29-32}$ In order to investigate the 
identity of this $870 \mathrm{~nm}$ band further, and thus validate this proposed mechanism, nanosecondmicrosecond TAS was subsequently performed.

\section{Nanosecond-microsecond transient absorption spectroscopy}

The pristine TDPP, pumped at $500 \mathrm{~nm}$, showed no signal at any probe wavelength on the timescales available (approximately $500 \mathrm{~ns}-10 \mathrm{~ms}$ ). In contrast, the TDPP:PC60BM film produced a large transient signal when pumped at the same wavelength. The TA spectrum of TDPP:PC60BM is shown in Figure 3a with a strong, narrow band appearing at $880 \mathrm{~nm}$ and a second distinct peak at $780 \mathrm{~nm}$. The position of this $880 \mathrm{~nm}$ band and its spectral shape bear a striking resemblance to the ps-TAS data, and thus is likely to represent the same transient species. The kinetics probed at $880 \mathrm{~nm}$ show clear mono-exponential kinetics with a lifetime of (14 \pm 1$) \mu \mathrm{s}$ (Figure $3 \mathrm{~b}$ ). This lifetime was invariant at low excitation densities, but at high excitation densities second order process begin to become evident. Such mono-exponential behaviour indicates a first order process such as triplet decay or charge carrier geminate recombination. The PC60BM triplet and radical anion can be discounted as their respective spectral shapes and positions are well known. ${ }^{33,34}$ Geminate recombination on timescales encompassing tens of microseconds is unlikely, and thus the most likely candidate for the $880 \mathrm{~nm}$ feature observed in the TA spectrum is the TDPP triplet, as suggested by the ps-TAS data. The loss of mono-exponential behaviour at higher excitation densities could therefore be assigned to triplet-triplet annihilation.

As with the ps-TAS, confirmation that the $880 \mathrm{~nm}$ peak can be assigned to a TDPP species rather than a fullerene transient species was provided by measuring the TA spectrum of TDPP:PC70BM. This spectrum, reported in Figure 3c, was virtually identical to that of TDPP:PC60BM, clearly showing that both the 880 and $780 \mathrm{~nm}$ peaks can be assigned to a TDPP transient species (both the radical anion and triplet state absorbance of PC70BM are distinctly red-shifted compared to those of PC60BM $\left.{ }^{27}, 28,35\right)$. The TDPP:PC70BM decay dynamics, shown in Figure 3d, could be fitted to a monoexponential decay with a lifetime of $(20 \pm 1) \mu \mathrm{s}$ : a slightly longer lifetime than that observed for TDPP:PC60BM. Again, this is tentatively assigned to the TDPP triplet, but additional confirmation is required. It should be noted that a prior study by Karsten et al. ${ }^{24}$ used triplet sensitisation to generate DPP triplets in a fullerene-DPP-fullerene triad in solution, where photoinduced absorption showed the resultant DPP triplet to have peaks at 860 and $775 \mathrm{~nm}$ - similar to those observed in this work. This close correlation, despite the differences in molecular structure and phase, is further evidence that the TDPP transient species observed here is the triplet state.

In order to confirm the assignment of the $880 \mathrm{~nm}$ peak to TDPP triplets, the decay kinetics were also measured under a pure oxygen atmosphere. It is expected that any triplets would be quenched by the triplet ground state of oxygen, thereby showing a shorter lifetime and smaller signal amplitude compared to the inert atmosphere. However, neither of these effects were observed: the kinetics were virtually identical under both argon and oxygen atmospheres for both blend films (Figure 3e and 3f). This would then suggest that the $880 \mathrm{~nm}$ peak belongs to the TDPP radical cation. However, it must be noted that the oxygen quenching mechanism can only take place if the TDPP triplet state is higher in energy than the first excited singlet state of oxygen. It has been suggested previously that DPP-based polymers have a very low-lying $T_{1}$ state, ${ }^{36}$ and it is therefore possible that the TDPP triplet energy is close to the oxygen quenching threshold of $0.98 \mathrm{eV}$. This would suggest that oxygen 
quenching may be very difficult in this system. However, previous results for a fullerene-DPPfullerene triad in solution show these DPP triplets to be sensitive to oxygen. ${ }^{23}$

It is thus not possible to conclude from these ns- $\mu$ s TAS results whether the $880 \mathrm{~nm}$ transient species being formed is the TDPP radical cation or triplet state. The microsecond lifetime of the monoexponential decay dynamics and close correlation with related molecules in the literature, ${ }^{24}$ however, are strongly suggestive of a triplet state. If the $880 \mathrm{~nm}$ feature observed in the TDPP/fullerene blend spectra is indeed from the TDPP triplet state, whereas no TDPP triplet is observed in a pristine TDPP film, the implication is that a direct intersystem crossing (ISC) mechanism cannot be responsible for the formation of the TDPP triplet. This is consistent with back recombination from a spin-mixing CT state, as proposed in the previous ps-TAS section. In order to address the ambiguity of the ns- $\mu$ S TAS results, and thereby confirm this mechanism, time-resolved EPR was performed.

Time-resolved electron paramagnetic resonance spectroscopy

TR-EPR experiments were performed for TDPP:PC70BM and its constituent components. PC70BM in solution produced a signal consistent with triplets formed via ISC that fully decay in $2 \mu$ s (Figure 4a). The signal presents a polarization pattern AAEE (where $E$ is emission and $A$ is enhanced absorption) and can be simulated with ZFS parameters $|D|=337 \mathrm{MHz}$ and $\mathrm{E}=0 \mathrm{MHz}$ and zero-field relative populations: $\mathrm{Px}: \mathrm{Py}: \mathrm{Pz}=0: 1: 1$, consistent with previously reported data for $\mathrm{C} 70$ monoadducts. ${ }^{37}$ The pristine TDPP film produces virtually no signal on the timescale of the technique, indicating a lack of either ISC triplets or photo-induced radicals (Figure 4b).

In contrast, TDPP:PC70BM ( $1: 2$ by weight, Figure $4 \mathrm{c}$ and $4 \mathrm{~d})$ produces a more complex, long-lived EPR signal. At early times (400-600 ns from the laser flash, Figure 4c), the EPR signal for the blend film can be assigned to two components. On the basis of the control experiments on PC70BM in solution discussed above, the central features can be attributed to PC70BM triplets populated via ISC and it reflects the high molar ratio of PC7OBM in the blend. On the basis of the ZFS and polarisation pattern respectively, the second broader component can be attributed to a triplet state formed on TDPP via radical pair recombination in high magnetic field. This is particularly apparent at long times of over $\sim 2 \mu \mathrm{s}$, when the PC70BM triplet has decayed (Figure 4d). Figure S2 (supporting information) reports the comparison of the two TR-EPR surfaces (field versus time) for PC70BM and TDPP:PC70BM (1:2 ratio) to illustrate the time dependence of the measured signals. The TR-EPR spectrum at later times presents polarization pattern AEEAAE, which is characteristic of selective population of the $\mathrm{T}_{0}$ sublevel (S-T $\mathrm{T}_{0}$ mixing) by charge recombination in the singlet-born weakly coupled radical pair at all orientations due to the conservation of the spin angular momentum in the recombination process. 38 The polarization pattern is indicative of a positive ZFS parameter $D$, which from simulation can be estimated to be $\mathrm{D}=+1550 \mathrm{MHz}$, together with a $|\mathrm{E}|=340 \mathrm{MHz}$. The EPR results are therefore entirely consistent with the proposed mechanism involving a TDPP:PC70BM charge transfer state that undergoes ultra-fast spin-mixing and recombination to yield the TDPP triplet state.

Discussion 
The proposed mechanism for the generation of the TDPP triplet state is shown in Figure 5, with the lifetimes of each state shown for the case of TDPP:PC70BM (1:2). It should be noted that similar spectroscopic results were obtained via fullerene excitation at $360 \mathrm{~nm}$, and also at other blend ratios. The ns- $\mu$ s spectra for 1.6:1 TDPP:fullerene films are shown in Figure S3, and are identical to those of the 1:2 blends, with clear formation of the TDPP triplet. This implies that the back recombination via a spin-mixing $\mathrm{CT}$ state is still present, and thus has little dependence on blend composition and morphology for this particular blend system. This was checked by acquiring AFM images for pristine TDPP, TDPP:PC60BM (1:2), TDPP:PC60BM (1.6:1), and TDPP:PC70BM (1:2) (Figure S4). It can be seen that the two 1:2 blends have evidence of significant order, with parallel structured domains apparent. In contrast, these parallel domains are absent in the 1.6:1 blend. Despite this large change in morphology, all blends produce spectroscopic evidence of triplet formation via back recombination from a spin-mixing CT state. As such, it can be concluded that energetics (the very low-lying TDPP triplet state) provide the greater driving force for the photophysics seen here, rather than morphology.

An interesting point to note is that the CT state spin-mixing and formation of the TDPP triplet occurs on picosecond timescales. The ps-TAS clearly show the growth of the TDPP triplet at $870 \mathrm{~nm}$, with the maximum population occurring at $30-40 \mathrm{ps}$. This is considerably faster than the spin-mixing and back recombination observed previously for other systems, such as the early nanosecond timescales measured for SilDT-2FBT/PC7OBM. ${ }^{7}$ It is unlikely that greater spin-orbit coupling is the primary cause of this: the largest atom in both TDPP and SilDT-2FBT is sulphur . If not enhanced spin-orbit coupling, one possible explanation for the fast spin-mixing is a very small - or even inverted ${ }^{39,40}$ singlet-triplet exchange energy in the TDPP:fullerene CT state. Since the exchange energy is directly related to the wavefunction overlap between the electron and hole, this would therefore imply a strongly separated electron and hole in the TDPP:fullerene CT state. ${ }^{41}$ The mechanism for spinmixing has been proposed to involve hyperfine interactions between the polaron electron spin and the surrounding proton spins. ${ }^{42}$ Hyperfine interactions are typically very weak, but can start to dominate when the two electron spins in the pair possess a large spatial separation, ${ }^{43}$ and therefore strong hyperfine interactions may also play a role in the ultra-fast spin-mixing. It has also been noted that ISC can occur very efficiently between ${ }^{1} \mathrm{CT}$ and a close lying triplet level of a single component (donor or acceptor), ${ }^{44,45}$ due to the spin flip coupling to a change in orbital angular momentum.

The lack of triplet formation in the pristine TDPP films, observed in both TAS and EPR results, suggests that no singlet fission occurs in this material, despite the energetics suitability. It should be noted, however, that the TDPP molecule studied in this work has a different side-chain to the TDPP in which singlet fission has previously been reported..$^{25}$ This may highlight the significant role side chains play on the electronic coupling required for singlet fission in small molecules.

The appearance of the TDPP triplet after photoexcitation in blends with fullerene indicates that this is the lowest lying excited state in the system - lower than that of the spin-mixing CT state. Indeed, the lack of oxygen quenching suggests that the TDPP triplet energy level is around or below $0.98 \mathrm{eV}$. There have been previous suggestions that DPP-based polymers have a very low-lying triplet state, ${ }^{36}$ ${ }^{46}$ and this study offers a strong support that this is indeed the case. Although spin-mixing CT states 
seem to be a common occurrence in polymer:fullerene systems (appearing in photovoltaic, ${ }^{47}$ OLED, ${ }^{48}$ and spintronic ${ }^{49}$ applications), they do not necessarily represent a loss mechanism in organic photovoltaics, provided there are no triplet states lower in energy. This therefore has important ramifications in terms of molecular design, as the formation of low-lying triplets must be avoided in order to eliminate a significant loss mechanism. Despite this, the direct observation of a small molecule / fullerene charge transfer state via the participating fullerene anion is very rare and will allow an unprecedented opportunity to study this elusive state in greater detail in the future.

Experimental

The TDPP was synthesised using a known literature procedure.50 Thin films of TDPP, TDPP:PC60BM, and TDPP:PC70BM (1:2 and 1.6:1 by weight) were prepared via spin-coating chloroform/odichlorobenzene (47:3 by volume) solutions onto glass substrates.

Nanosecond-microsecond TAS: Microsecond TAS on the above film samples was recorded using laser pulses ( $6 \mathrm{~ns}, 532 \mathrm{~nm}$, repetition rate $10 \mathrm{~Hz}$ ) from a Nd:YAG laser (Spectra-Physics, INDI-40-10) with a pump wavelength of $510 \mathrm{~nm}$, using pump intensities of $0.5-36 \mu \mathrm{cm}^{-2}$. The light output of a tungsten lamp (Bentham) was used as a probe and signals were recorded with Si and InGaAs photodiodes, housed in a preamplifier and an electronic filter (Costronics Electronics) connected to an oscilloscope and PC. Probe wavelengths were selected with a monochromator. The films were kept under an argon atmosphere to prevent film degradation, while an oxygen atmosphere was trialled to check for triplet quenching.

Picosecond TAS: Film samples under inert nitrogen atmosphere were excited with $510 \mathrm{~nm}, 2-12 \mu \mathrm{J}$ $\mathrm{cm}^{-2}$ pulses, generated by a commercially available optical parametric amplifier TOPAS (Light conversion) pumped by a Solstice Ti:Sapphire regenerative amplifier (Newport Ltd). Changes in the optical density of the films induced by the laser excitation were probed with a second broadband pulse $(830-1450 \mathrm{~nm})$ generated in a sapphire crystal. A HELIOS (Ultrafast systems) transient absorption spectrometer was used for recording the dynamics of the transient absorption spectra up to $6.5 \mathrm{~ns}$ with an average $200 \mathrm{fs}$ instrument response function.

Time-resolved EPR: A Bruker E580 pulsed EPR spectrometer operating at $~ 9.6 \mathrm{GHz}$ and equipped with a Bruker ER4118X-MD5 dielectric resonator was used to record the TR-EPR spectra solution (PC70BM, 100 $\mu \mathrm{M}$ ) and thin film (TDPP and TDPP:PC70BM (1:2) blend). Spectra were recorded in direct detection mode without magnetic field modulation. Hence they show characteristic enhanced absorptive $(A)$ and emissive (E) features, as indicated in the reported spectra. A Surelite broadband OPO system within the operating range $410-680 \mathrm{~nm}$, pumped by a Surelite I-20 Q-switched Nd:YAG laser with 2nd and 3rd harmonic generators ( $20 \mathrm{~Hz}$, pulse length of $5 \mathrm{~ns}$ ) was used to achieve a pulsed laser excitation at an appropriate wavelength optimized on the signal intensity, with the energy at the sample approximately $10 \mathrm{~mJ}$ per pulse. A cryogen-free cryostat from Cryogenic Itd and a Lake Shore temperature controller (model 350) were used to cool the sample and maintain the temperature at $50 \mathrm{~K}$. EPR samples were prepared as described above and sealed under vacuum in quartz tubes (outer diameter $5 \mathrm{~mm}$ ). TR-EPR spectra were simulated using the EasySpin toolbox 51 in $M^{M A T L A B}{ }^{\text {TM }}$ to extract ZFS parameters - D and $\mathrm{E}-$ and sublevel populations ( $\left.\mathrm{Px}, \mathrm{Py}, \mathrm{Pz}\right)$. The simulation of the TR-EPR spectrum of PC70BM in solution took as initial parameters literature data 
for $\mathrm{C70}$ monoadducts.37 The simulation of the DPP:PC70BM thin film considered the ZFS parameters $\mathrm{D}$ and $\mathrm{E}$ to be both positive. An isotropic $\mathrm{g}$ value equal to the free electron $\mathrm{g}$ value $(\mathrm{gx}=\mathrm{gy}=\mathrm{gz}=$ 2.0023) was used in all simulations.

Conclusions

Small molecule TDPP:fullerene bulk heterojunction blend films were examined with TAS and TR-EPR, with specific focus on transient absorption spectral features in the near-infrared region. This spectroscopic study establishes a baseline of photophysical behaviour that will create a foundation for spectroscopic investigations into more complex DPP-based molecules, commonly used in organic electronic applications.

It was directly observed that a TDPP:fullerene charge transfer state forms on ultrafast timescales, shorter than the instrument resolution ( $<100 \mathrm{fs}$ ). This $\mathrm{CT}$ state undergoes unusually fast spin-mixing and geminate recombination to form the TDPP triplet state, the lowest energy excited state in the system. The presence of the CT state was directly inferred via a clearly identifiable fullerene anion transient absorption spectral signature with a monoexponential lifetime of approximately $200-500$ ps (depending on the identity of the fullerene). This is one of the first times an intermolecular small molecule/fullerene charge transfer state has been observed spectroscopically. This presence of the TDPP:fullerene CT state and overall photophysical mechanism was confirmed using TR-EPR spectroscopy, which explicitly shows that the TDPP triplet has been formed through a spin-mixing CT state mechanism rather than by direct ISC from the excited single state.

Conflicts of interest

There are no conflicts to declare.

Acknowledgements

We would like to thank Professor James Durrant for the use of his ps-TAS system at Imperial College London, and Caroline Nowicka-Dylag for measuring the AFM images. TMC would like to acknowledge support from EPSRC project EP/N026411/1. HB would like to acknowledge ERC grant CONTREX.

Author contributions.

The TDPP was synthesised by AL, supervised by HB. Film samples were fabricated by NL and JS under the supervision of TMC. TAS was performed by NL, JS, and TMC, with the data analysed by NL and TMC. EPR was performed by ES and the data analysed by ES and CWMK. The paper was written by TMC with assistance from ES and CWMK. The study was conceived by TMC and supervised by TMC, $\mathrm{HB}$, and CWMK.

Notes and references

1 S. Qu and H. Tian, Chem. Commun., 2012, 48, 3039.

2 W. Li, K. H. Hendriks, M. M. Wienk and R. A. J. Janssen, Acc. Chem. Res., 2016, 49, 78.

3 K. H. Hendriks, G. H. L. Heintges, V. S. Gevaerts, M. M. Wienk and R. A. J. Janssen, Angew. Chem. Int. Edit., 2013, 52, 8341. 
4 R. S. Ashraf, I. Meager, M. Nikolka, M. Kirkus, M. Planells, B. C. Schroeder, S. Holliday, M. Hurhangee, C. B. Nielsen, H. Sirringhaus and I. McCulloch, JACS, 2015, 137, 1314.

5 H. Choi, S.-J. Ko, T. Kim, P.-O. Morin, B. Walker, B. H. Lee, M. Leclerc, J. Y. Kim and A. J. Heeger, Adv. Mater., 2015, 27, 3318.

6 Y. Kan, C. Liu, L. Zhang, K. Gao, F. Liu, J. Chen and Y. Cao, J. Mater. Chem. A, 2016, 4, 14720.

7 S. D. Dimitrov, S. Wheeler, D. Niedzialek, B. C. Schroeder, H. Utzat, J. M. Frost, J. Yao, A. Gillett, P. S. Tuladhar, I. McCulloch, J. Nelson and J. R. Durrant, Nat. Commun., 2015, 6, 6501.

8 W. Chang, D. N. Congreve, E. Hontz, M. E. Bahlke, D. P. McMahon, S. Reineke, T. C. Wu, V. Bulović, T. Van Voorhis and M. A. Baldo, Nat. Commun., 2015, 6, 6415.

9 S. L. Bayliss, N. C. Greenham, R. H. Friend, H. Bouchiat and A. D. Chepelianskii, Nat. Commun., 2015, 6, 8534.

10 T. Clarke, A. Ballantyne, F. Jamieson, C. Brabec, J. Nelson and J. Durrant, Chem. Commun., 2009, 1, 89.

11 M. P. Eng, P. R. F. Barnes and J. R. Durrant, J. Phys. Chem. Lett., 2010, 1, 3096.

12 J. Guo, H. Ohkita, H. Benten and S. Ito, JACS, 2009, 131, 16869.

13 J. Guo, H. Ohkita, S. Yokoya, H. Benten and S. Ito, JACS, 2010, 132, 9631.

14 H. Ohkita, S. Cook, Y. Astuti, W. Duffy, S. Tierney, W. Zhang, M. Heeney, I. McCulloch, J. Nelson, D. D. C. Bradley and J. R. Durrant, JACS, 2008, 130, 3030.

15 A. Ruseckas, M. Theander, M. R. Andersson, M. Svensson, M. Prato, O. Inganäs and V. Sundstrom, Chem. Phys. Lett., 2000, 322, 136.

16 A. Saeki, S. Seki, Y. Koizumi and S. Tagawa, J. Photoch. Photobio. A, 2007, 186, 158.

17 S. Shoaee, M. P. Eng, E. Espildora, J. L. Delgado, B. Campo, N. Martin, D. Vanderzande and J. R. Durrant, Energ. Environ. Sci., 2010, 3, 971.

18 S. Subramaniyan, H. Xin, F. S. Kim, S. Shoaee, J. R. Durrant and S. A. Jenekhe, Adv. Energy Mat., 2011, 1, 854.

19 S. Yamamoto, H. Ohkita, H. Benten and S. Ito, J. Phys. Chem. C, 2012, 116, 14804.

20 J. Nelson, Phys. Rev. B, 2003, 67, 155209.

21 H. Liu, H. Jia, L. Wang, Y. Wu, C. Zhan, H. Fu and J. Yao, Phys. Chem. Chem. Phys., 2012, 14, 14262.

22 P. E. Hartnett, S. M. Dyar, E. A. Margulies, L. E. Shoer, A. W. Cook, S. W. Eaton, T. J. Marks and M. R. Wasielewski, Chem. Sci., 2015, 6, 402.

23 N. Banerji, M. Wang, J. Fan, E. S. Chesnut, F. Wudl and J.-E. Moser, J. Mater. Chem., 2012, 22,13286 .

24 B. P. Karsten, R. K. M. Bouwer, J. C. Hummelen, R. M. Williams and R. A. J. Janssen, Photochem. Photobio. Sci., 2010, 9, 1055. 
25 P. E. Hartnett, E. A. Margulies, C. M. Mauck, S. A. Miller, Y. Wu, Y.-L. Wu, T. J. Marks and M. R. Wasielewski, J. Phys. Chem. B, 2016, 120, 1357.

26 T. M. Clarke, A. M. Ballantyne, J. Nelson, D. D. C. Bradley and J. R. Durrant, Adv. Func. Mater., 2008, 18, 4029

27 D. R. Lawson, D. L. Feldhiem, C. A. Foss, P. K. Dorhout, C. M. Elliott, C. R. Martin and B. Parkinson, J. Phys. Chem., 1992, 96, 7175.

28 A. Sperlich, M. Liedtke, J. Kern, H. Kraus, C. Deibel, S. Filippone, J. L. Delgado, N. Martín and V. Dyakonov, phys. status solidi - R, 2011, 5, 128.

29 D. Moghe, P. Yu, C. Kanimozhi, S. Patil and S. Guha, Charge transfer complex states in diketopyrrolopyrrole polymers and fullerene blends: Implications for organic solar cell efficiency, 2011.

30 T. Hahn, S. Tscheuschner, C. Saller, P. Strohriegl, P. Boregowda, T. Mukhopadhyay, S. Patil, D. Neher, H. Bässler and A. Köhler, J. Phys. Chem. C, 2016, 120, 25083.

31 S. Few, J. M. Frost, J. Kirkpatrick and J. Nelson, J. Phys. Chem. C, 2014, 118, 8253.

32 M.-F. Falzon, A. P. Zoombelt, M. M. Wienk and R. A. J. Janssen, Phys. Chem. Chem. Phys., $2011,13,8931$.

33 S. Cook, H. Ohkita, J. R. Durrant, Y. Kim, J. J. Benson-Smith, J. Nelson and D. D. C. Bradley, Appl. Phys. Lett., 2006, 89, 101128.

34 H. Ohkita and S. Ito, Polymer, 2011, 52, 4397.

35 P. C. Y. Chow, S. Albert-Seifried, S. Gélinas and R. H. Friend, Adv. Mater., 2014, 26, 4851.

36 Y. W. Soon, H. Cho, J. Low, H. Bronstein, I. McCulloch and J. R. Durrant, Chem. Commun., 2013, 49, 1291.

37 L. Franco, A. Toffoletti and M. Maggini, Phys. Chem. Chem. Phys., 2012, 14, 14358.

38 P. J. Hore, Advanced EPR, Elsevier Science Publishers, B.V. , Amsterdam, 1989.

39 W. Li, Y. Pan, R. Xiao, Q. Peng, S. Zhang, D. Ma, F. Li, F. Shen, Y. Wang, B. Yang and Y. Ma, Adv. Func. Mater., 2014, 24, 1609.

40 S. Difley, D. Beljonne and T. Van Voorhis, JACS, 2008, 130, 3420.

41 W. Chang, D. N. Congreve, E. Hontz, M. E. Bahlke, D. P. McMahon, S. Reineke, T. C. Wu, V. Bulović, T. Van Voorhis and M. A. Baldo, 2015, 6, 6415.

42 V. V. Mkhitaryan and V. V. Dobrovitski, Phys. Rev. B, 2017, 95, 214202.

43 S. Oviedo-Casado, A. Urbina and J. Prior, Scientific Reports, 2017, 7, 4297.

44 F. B. Dias, J. Santos, D. R. Graves, P. Data, R. S. Nobuyasu, M. A. Fox, A. S. Batsanov, T. Palmeira, M. N. Berberan-Santos, M. R. Bryce and A. P. Monkman, Advanced Science, 2016, 3, 1600080.

45 B. T. Lim, S. Okajima, A. K. Chandra and E. C. Lim, Chem. Phys. Lett., 1981, 79, 22. 
46 J. R. Ochsmann, D. Chandran, D. W. Gehrig, H. Anwar, P. K. Madathil, K.-S. Lee and F. Laquai, Macro. Rapid Commun., 2015, 36, 1122.

47 Z. Xu and B. Hu, Adv. Func. Mater., 2008, 18, 2611.

48 W. Li, Y. Pan, L. Yao, H. Liu, S. Zhang, C. Wang, F. Shen, P. Lu, B. Yang and Y. Ma, Advanced Optical Materials, 2014, 2, 892.

49 J. Wang, A. Chepelianskii, F. Gao and N. C. Greenham, 2012, 3, 1191.

50 C.-W. Ge, C.-Y. Mei, J. Ling, F.-G. Zhao, H.-J. Li, L. Liang, J.-T. Wang, J.-C. Yu, W. Shao, Y.-S. Xie and W.-S. Li, J. Polym. Sci. A1, 2014, 52, 2356.

51 S. Stoll and A. Schweiger, J. Magn. Reson., 2006, 178, 42.

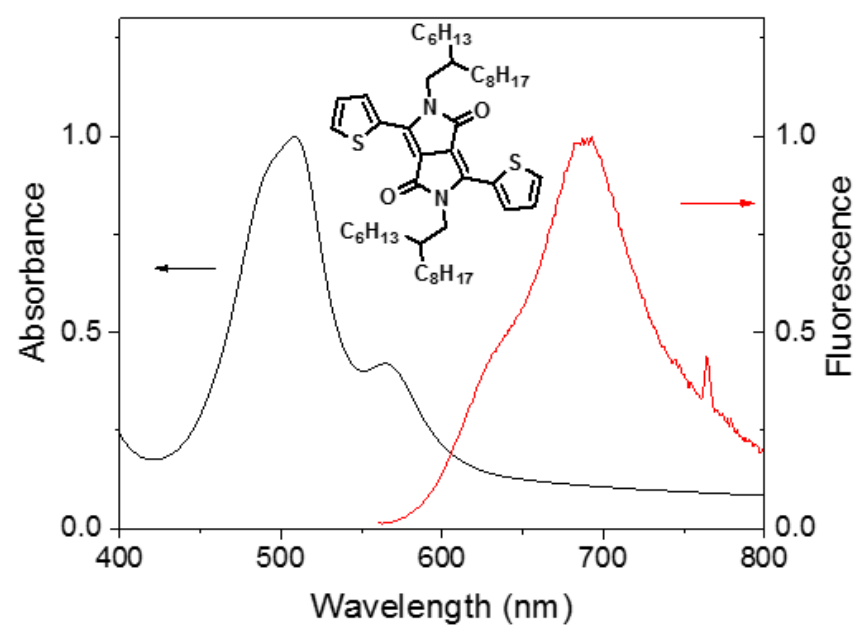

Figure 1. The normalised steady state absorbance and fluorescence spectra of pristine TDPP films, with the molecular structure shown in the inset. 

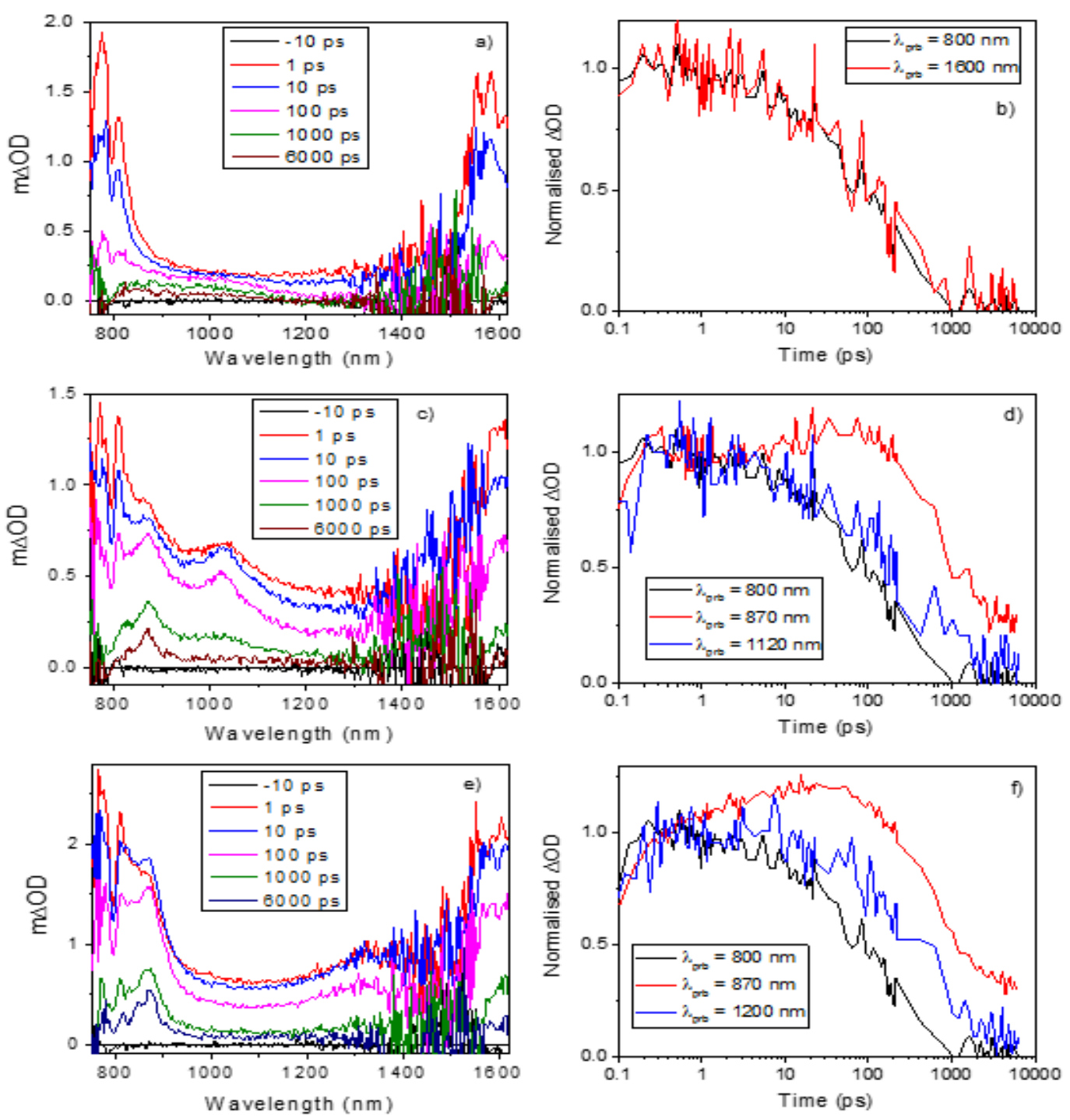

Figure 2. The picosecond transient absorption spectra and kinetics respectively as a function of probe wavelength for (a, b) a pristine TDPP film, (c, d) a TDPP:PC ${ }_{60} B M$ film (1:2 by weight), and (e, f) a TDPP:PC ${ }_{70} B M$ film (1:2 by weight). A pump wavelength of $510 \mathrm{~nm}$ was used, and excitation densities of 2.7 and $7.3 \mu \mathrm{Jm}^{-2}$ were used for the kinetics and spectra respectively. 

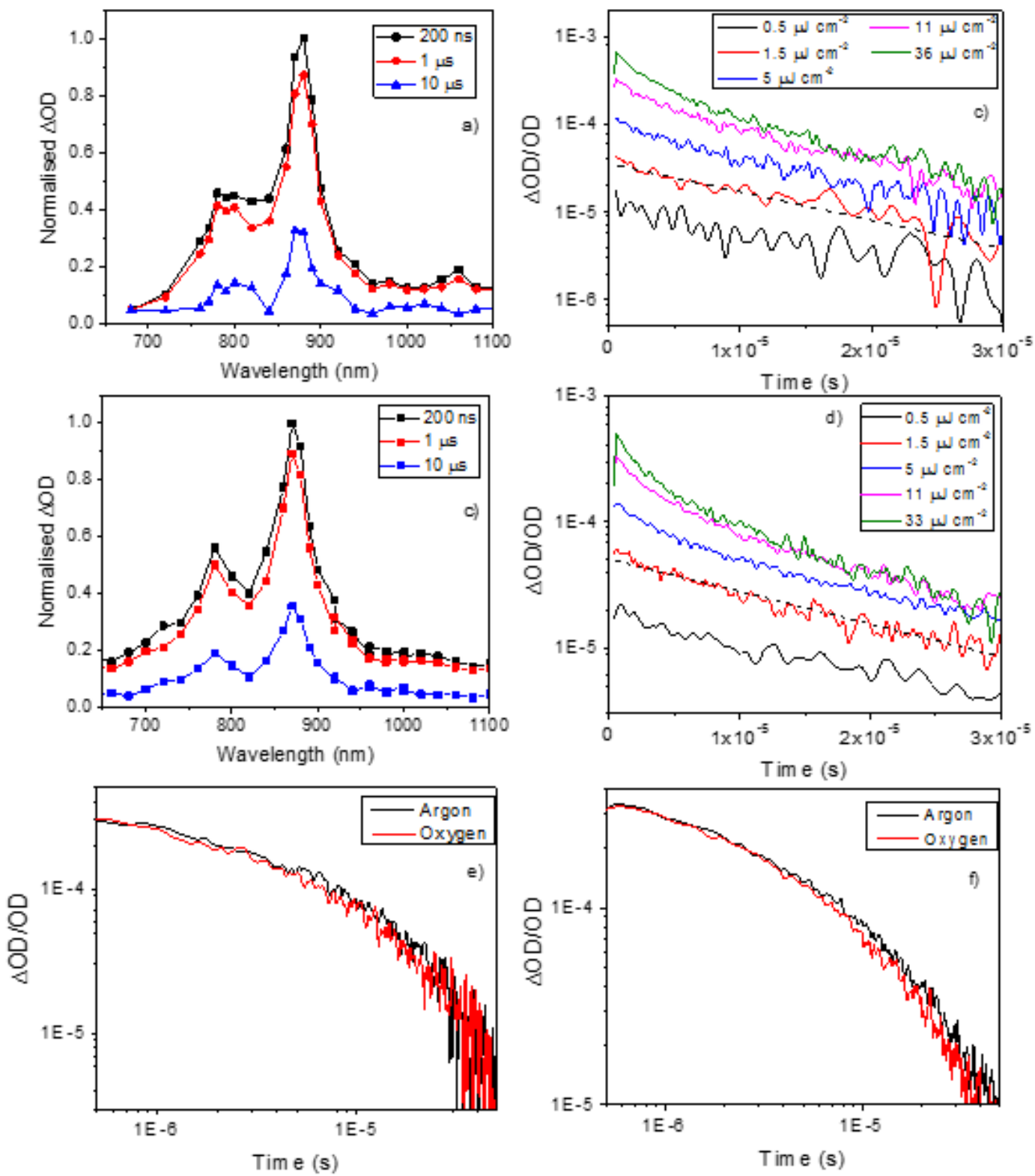

Figure 3. The $\mu$ s transient absorption spectra films of (a) TDPP:PC ${ }_{60} \mathrm{BM}$ and (b) TDPP:PC ${ }_{70} \mathrm{BM}$, both 1:2 by weight, using $510 \mathrm{~nm}$ and $36 \mu \mathrm{cm}{ }^{-2}$ excitation. The corresponding decay kinetics as a function of excitation density for (c) TDPP:PC 60 BM and (d) TDPP:PC $\mathrm{C}_{70} \mathrm{BM}$, both 1:2 by weight, using $510 \mathrm{~nm}$ excitation and a probe wavelength of $875 \mathrm{~nm}$. The dependence on oxygen is shown for (e) TDPP:PC 60 BM and (f) TDPP:PC $70 \mathrm{BM}$, both $1: 2$ by weight, using $510 \mathrm{~nm}, 11 \mu \mathrm{Jm}^{-2}$ excitation and a probe wavelength of $875 \mathrm{~nm}$. 


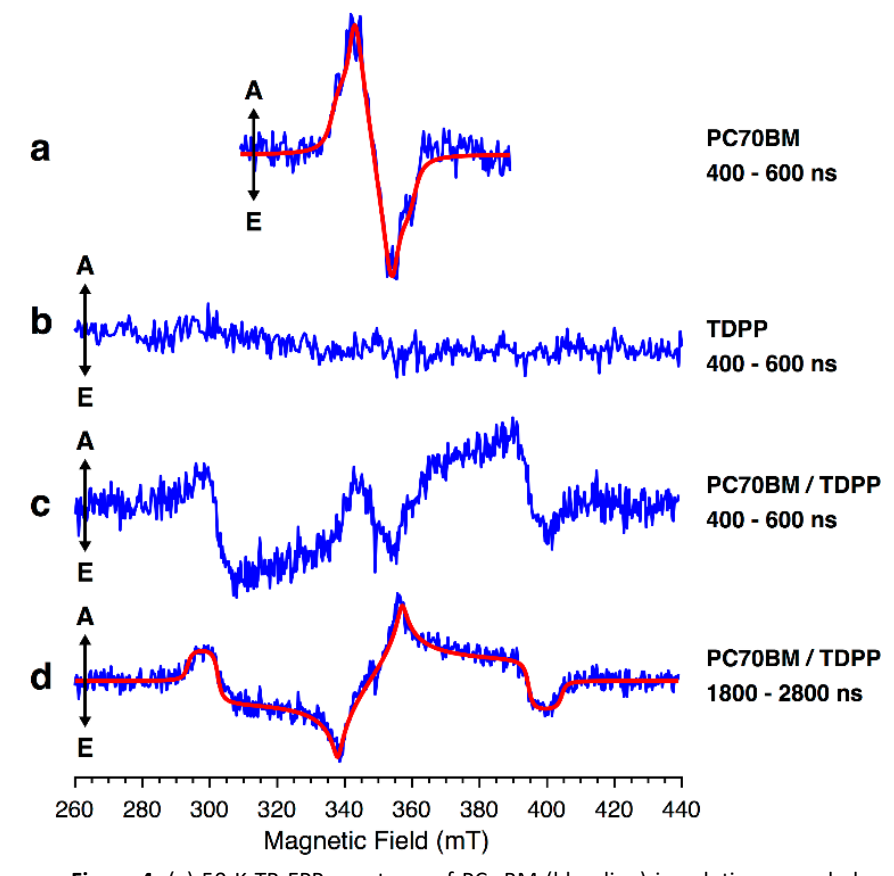

Figure 4. (a) $50 \mathrm{~K}$ TR-EPR spectrum of $\mathrm{PC}_{70} \mathrm{BM}$ (blue line) in solution recorded after 300-500 ns from the laser pulse. The $\mathrm{PC}_{70} \mathrm{BM}$ spectrum can be simulated with ZFS parameters $\mathrm{D}=337 \mathrm{MHz},|\mathrm{E}|=0$ and relative populations $\mathrm{Px}: \mathrm{Py}: \mathrm{Pz}=$ 0:1:1 (red line). (b) $50 \mathrm{~K}$ TR-EPR spectrum of a pristine TDPP film recorded after 400-600 ns from the laser pulse. (c) $50 \mathrm{~K}$ TR-EPR spectrum for the TDPP:PC ${ }_{70} \mathrm{BM}$ $1: 2$ blend film at early times (400-600 ns after the laser flash), where two contributions are distinguishable: a broad triplet and a narrower feature reminiscent of the $\mathrm{PC}_{70} \mathrm{BM}$ triplet. (d) $50 \mathrm{~K}$ TR-EPR spectrum for the TDPP:PC70BM 1:2 blend film at later times (1800-2800 ns after the laser flash): only the recombination triplet on TDPP is visible. The TDPP:PC ${ }_{70} \mathrm{BM}$ spectrum can be simulated with ZFS parameters $\mathrm{D}=+1550 \mathrm{MHz},|\mathrm{E}|=340 \mathrm{MHz}$ and assuming that only the $T_{0}$ sublevel in high-field is populated via the charge transfer state mechanism.

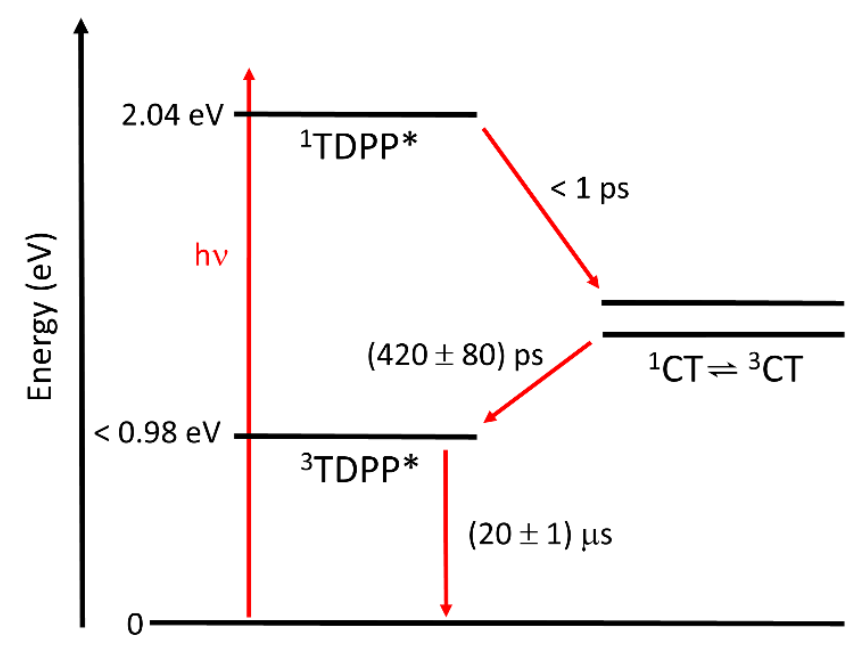

Figure 5. State diagram for the proposed model of TDPP triplet generation in TDPP:PC ${ }_{70} \mathrm{BM}$ films. Note that while the same mechanism applies for TDPP:PC ${ }_{60} \mathrm{BM}$, the associated kinetic parameters differ. It is assumed here that the geminate recombination lifetime of $(420 \pm 80)$ ps observed for the CT state solely produces TDPP triplets, but some geminate recombination to the ground state is also possible. 